\title{
NONLINEAR ANALYSIS AND QUASICONFORMAL MAPPINGS FROM THE PERSPECTIVE OF PDES
}

\author{
TADEUSZ IWANIEC \\ Department of Mathematics, Syracuse University \\ Syracuse, New York 13244, U.S.A. \\ E-mail: tiwaniec@mailbox.syr.edu
}

\section{Contents}

Introduction 119

1. Quasiregular mappings 120

2. The Beltrami equation 121

3. The Beltrami-Dirac equation 122

4. A quest for compactness 124

5. Sharp $L^{p}$-estimates versus variational integrals 125

6. Very weak solutions 128

7. Nonlinear commutators 129

8. Jacobians and wedge products 131

9. Degree formulas 134

References 136

Introduction. The general trend of the geometric function theory in $\mathbb{R}^{n}$ is to generalize certain topological aspects of the analytic functions of one complex variable. The category of mappings that one usually considers in higher dimensions are the mappings with finite distortion, thus, in particular, quasiconformal and quasiregular mappings. This program, whose origin can be traced back to the works of M. A. Lavrentiev (1938), L. V. Ahlfors (1954), F. W. Gehring (1961), J. Väisälä (1961) and Y. Reshetnyak (1966), was held by an important school of Finnish geometers in the 1970's, led by O. Martio, S. Rickman and J. Väisälä. For a recent account see [Ri] and [Vu].

But in this drive towards generalizations of analytic functions, one aspect has been quite neglected. This is the fact that the mappings in question solve important first order systems of PDEs analogous in many respects to the Cauchy-Riemann equations.

1991 Mathematics Subject Classification: Primary 46C20; Secondary 32G81.

Research supported in part by NSF grant DMS-9706611.

The paper is in final form and no version of it will be published elsewhere. 
The solutions of these systems can be viewed as "absolute" minimizers of certain energy functionals. It is striking how tight the connection is between quasiregular mappings and the development of the nonlinear elasticity theory whose mathematical principles were already formulated by S. S. Antman [A] and J. Ball [B] in 1976-77. Roughly speaking, the theory of elasticity studies mappings, referred to as deformations of elastic bodies, which minimize the so-called stored energy functionals. These functionals are not always convex and the deformations need not be quasiconformal but the governing PDEs are much the same. The Jacobian determinant, in particular, has been subjected to a great deal of investigation. We shall give a brief account of this study together with some generalizations concerning wedge products of closed differential forms. The latter arise naturally in the theory of compensated compactness by F. Murat [Mu1] and L. Tartar [Ta].

A fruitful idea when studying quasiconformal mappings is to view them as conformal with respect to certain measurable Riemannian metric structures. This is a view which we shall largely adopt and extend to manifolds suitably. It is a deep result of D. Sullivan that all topological $n$-manifolds, $n \neq 4$, admit quasiconformal structures. As instances, following S. Donaldson and D. Sullivan [DS] one can develop a measurable Yang-Mills theory and analogues of the Atiyah-Singer index theory. A clear need has emerged to consolidate these approaches. This program stays beyond the confines of the present lectures, though we hope to call on it in the nearest future.

One key for the understanding and creation of new types of PDEs for mappings of finite distortion lies in the constant development and refinement of the de Rham cohomology theory which includes nonlinear boundary value problems on manifolds. We dignify these equations by calling them the Hodge systems. In this way all the equations relevant to quasiconformal mappings can be represented in a concise and elegant manner by using differential forms. In a way, this is a statement of my recent approach to quasiconformal analysis and nonlinear PDEs. It is important to realize that this task required a supreme effort on the part of my recent students and scholars. I would like to give special thanks to Chad Scott, Len Budney, Ray DeCampo, Luigi Greco and Bianca Stroffolini. I hope that my enthusiasm for certain ideas will infiltrate the minds of a few new students. That is the best we could get in return.

Our lecture will begin with a brief overview of the theory pointing out some of the highlights and new terminology. It is intended to spark subsequent questions; many of them are new and appear here for the first time.

1. Quasiregular mappings. It is advantageous to discuss the quasiregular mappings in the context of the Riemannian manifolds. This more general setting provides a suitable framework for better understanding the forthcoming PDEs in the Euclidean domains as well.

Let $X$ and $Y$ be $C^{\infty}$-smooth oriented Riemannian manifolds of dimension $n \geq 2$. We do not reserve any particular notation for the metric tensors on $X$ and $Y$ as these tensors are fixed for the duration of our discussion. They appear implicitly when we speak of norms and inner products of vectors in the tangent spaces $T_{x} X$ and $T_{y} Y$. The volume forms on $X$ and $Y$, denoted by $d x$ and $d y$, will be the ones induced by the orientation 
and the metric tensors. We shall consider mappings $f: X \rightarrow Y$ in the Sobolev space $W^{1, p}(X, Y)$. Be aware of nuances concerning this space; $C^{\infty}(X, Y)$ need not be dense in $W^{1, p}(X, Y)$ when $1 \leq p<n$. This case was settled by $\mathrm{F}$. Bethuel only eight years ago [Be], confront it with Question 11 here. The differential $D f(x): T_{x} X \rightarrow T_{y} Y$, $y=f(x)$, is defined almost everywhere. Most of the time we shall assume that the Jacobian determinant $J(x, f)=\operatorname{det} D f(x)$ is nonnegative and refer to such mappings as orientation preserving. The linear distortion function $K(, f): X \rightarrow[1, \infty]$ is then defined by

$$
K(x, f)=\frac{\max \left\{|D f(x) \xi| ; \xi \in T_{x} X,|\xi|=1\right\}}{\min \left\{|D f(x) \xi| ; \xi \in T_{x} X,|\xi|=1\right\}} .
$$

Here at some points the differential $D f(x)$ may not be defined or the indefinite quotient $\frac{0}{0}$ may occur and, as a convention, we understand $K(x, f)$ to be equal to 1 in such cases.

Definition 1. An orientation preserving mapping $f \in W^{1, p}(X, Y)$ is said to have finite distortion if $K(x, f)<\infty$ a.e. It is $K$-quasiregular $(1 \leq K<\infty)$ if its maximal dilatation $K(f)=\operatorname{esssup}\{K(x, f) ; x \in X\}$ does not exceed $K$. A homeomorphism which is $K$-quasiregular is called $K$-quasiconformal.

Needless to say the natural Sobolev exponent here is the dimension of the manifolds; mappings of finite distortion in $W^{1, n}(X, Y)$ are continuous [VG]. The case $1 \leq p<n$, having a great effect on the removability of singularities [I 1], [IM 1], has to be treated seriously as well. The removability questions were the subject of my prior survey articles and we will not go into it here [I 2,3], see also [IMNS]. Strangely, the case below the natural exponent takes great cleverness to deal with; it leads to the so-called very weak solutions of PDEs [IS2], [L]. It is in this context that the $L^{p}$-theory comes into its own and gives rise to a number of problems. The classical references to the general theory of quasiregular mappings are: $[\mathrm{V}],[\mathrm{Re} 2],[\mathrm{BI}],[\mathrm{HKM}]$ and $[\mathrm{Ri}]$.

2. The Beltrami equation. Since tangent spaces are furnished with an inner product it makes sense to speak of the transposed differential $D^{t} f(x): T_{y} Y \rightarrow T_{x} X$. In nonlinear elasticity, the bundle endomorphism $C=D^{t} f D f: T X \rightarrow T X$ is referred to as the right Cauchy-Green strain tensor. In many instances this parallels the distortion tensor $G: T X \rightarrow T X$ in quasiconformal theory defined by: $G(x)=J(x, f)^{-\frac{2}{n}} C(x)$ if $D f(x)$ exists and is nonsingular and $G(x)=I$ otherwise. The fundamental interplay between mappings of finite distortion and nonlinear PDEs is established via the Beltrami equation

$$
D^{t} f(x) D f(x)=J(x, f)^{\frac{2}{n}} G(x) .
$$

That $f$ solves this equation is, of course, a tautology. Nevertheless, we are hoping to investigate mappings of finite distortion by viewing them as solutions of this PDE and by respecting the ellipticity condition

$$
K^{2-2 n}|\xi|^{2 n} \leq\langle G(x) \xi, \xi\rangle^{n} \leq K^{2 n-2}|\xi|^{2 n}
$$

with $K=K(x, f) \geq 1$, and $\xi \in T_{x} X$. 
We shall regard $G$ as a new measurable conformal structure on $X$, so we can legitimately quote $f$ as conformal with respect to this structure. The complexities of the measurable conformal structures are formidable indeed, due mainly to the difficulty in defining the curvature tensor. On the other hand, topologically interesting mappings (branch sets) occur only in the presence of measurable structures. And here is the vital point: certain ideas of conformal geometry can be carried over to the measurable Riemannian structures without the necessity of differentiating the metric tensor. This simple observation is at the heart of our method. In order to gain some benefit from the Beltrami equation we would have to derive new equations from it.

3. The Beltrami-Dirac equation. We shall take a little time to trace our earlier investigations [IM1], [I], [Ma] that led us to the governing PDEs. Let $\wedge^{l} X, l=0,1, \ldots, n$, denote the $l$ th-exterior power of the cotangent bundle over $X$. Sections of $\wedge^{l} X$, denoted by $\Gamma\left(\wedge^{l} X\right)$, are the $l$-forms on $X$. Continuing in this fashion the symbols $C^{\infty}\left(\wedge^{l} X\right), L^{p}\left(\wedge^{l} X\right)$ $W^{1, p}\left(\wedge^{l} X\right)$, etc. for corresponding subspaces of $\Gamma\left(\wedge^{l} X\right)$ become self-explanatory.

The exterior derivative $d: C^{\infty}\left(\wedge^{l-1} X\right) \rightarrow C^{\infty}\left(\wedge^{l} X\right)$ and its formal adjoint $d^{\star}=$ $(-1)^{n l+1} \star d \star: C^{\infty}\left(\wedge^{l} X\right) \rightarrow C^{\infty}\left(\wedge^{l-1} X\right)$ are the fundamental differential operators on forms. Here $\star: \wedge^{l} X \rightarrow \wedge^{n-l} X$ stands for the Hodge star duality operator. More natural domains for $d$ and $d^{\star}$ will become perfectly clear in the sequel. One more space of concern to us consists of harmonic fields on the target manifold $Y$, denoted by $\mathcal{H}^{l}(Y)=\{\theta \in$ $\left.C^{\infty}\left(\wedge^{l} Y\right), d \theta=d^{\star} \theta=0\right\}$.

We can now recall a K-quasiregular mapping $f \in W^{1, n}(X, Y)$. Its distortion tensor $G: T X \rightarrow T X$ induces a symmetric positive definite bundle automorphism $G_{\#}: \wedge^{l} X \rightarrow$ $\wedge^{l} X$, that is: $K^{-a}|\xi|^{2 n} \leq\left\langle G_{\#} \xi, \xi\right\rangle^{n} \leq K^{a}|\xi|^{2 n}, a=2 l(n-l)$, for all $\xi \in \Gamma\left(\wedge^{l} X\right)$. This is just another way of saying that $f$ is $K$-quasiregular. In local coordinates, $G_{\#}(x)$ is represented by a matrix of size $\left(\begin{array}{l}n \\ l\end{array}\right) \times\left(\begin{array}{l}n \\ l\end{array}\right)$ whose entries are the $l \times l$-minors of $G(x)$.

Associated with a quasiregular mapping $f \in W^{1, n}(X, Y)$ is the pullback of $l$-forms $f^{\#}: C^{\infty}\left(\wedge^{l} Y\right) \rightarrow L^{p}\left(\wedge^{l} X\right)$ with $p=\frac{n}{l}$. Worth noting is the commutation rule $d \circ f^{\#}=$ $f^{\#} \circ d$. An interplay between the Hodge star operator and the pullback is essential for the derivation of the governing PDEs. To this end, let us fix an arbitrary harmonic field $\theta \in \mathcal{H}^{l}(Y)$. By virtue of the commutation rule, the pullbacks $f^{\#}(\theta)$ and $f^{\#}(\star \theta)$ are closed forms on $X$. With the aid of the Hodge-de Rham theory we can express them (locally) as:

$$
f^{\#}(\theta)=d u, \quad d^{\star} u=0 \quad \text { and } \quad \star f^{\#}(\star \theta)=d^{\star} v, \quad d v=0 .
$$

Now, the differential forms $u \in W^{1, p}\left(\wedge^{l-1} X\right)$ and $v \in W^{1, q}\left(\wedge^{l+1} X\right), \frac{1}{p}+\frac{1}{q}=1$, will be the unknowns of our equations. One can look at them as multi-dimensional analogues of the real and imaginary part of a holomorphic function. First we take up the case of a conformal mapping $f \in W^{1, l}(X, Y)$ in even dimension $n=2 l$. The equations obtained for $u$ and $v$ are extremely simple:

$$
d u=d^{\star} v \quad \text { and } \quad d^{\star} u=d v=0 .
$$

In particular, both $u$ and $v$ are harmonic forms, consequently $C^{\infty}$-smooth. 
It hardly matters how we choose the underlying field $\theta \in \mathcal{H}^{l}(Y)$; our equations depend purely upon the differential. We indulge ourselves by putting on stage some simple ones. Fix orthonormal bases in $T_{x} X$ and $T_{y} Y, y=f(x)$, then represent $D f(x): T_{x} X \rightarrow T_{y} Y$ as a square $2 l \times 2 l$-matrix. We infer from (5) the following relations:

$$
D f(x)=\left[\begin{array}{ll}
A(x) & B(x) \\
C(x) & D(x)
\end{array}\right], \quad\left\{\begin{array}{l}
\operatorname{det} A=\operatorname{det} D \\
\operatorname{det} B=(-1)^{l} \operatorname{det} C
\end{array}\right.
$$

where $A, B, C$ and $D$ are the $l \times l$-submatrices. Notice the resemblance to the CauchyRiemann system in $\mathbb{R}^{2}$. When the rows and columns are permuted in $D f(x)$, more linear relations between the $l \times l$-minors are obtained. In much the same way the general Beltrami equation (2) in dimension $n=2 l$ when lifted to the exterior bundle $\wedge^{l} X$ involves linear equations with measurable coefficients for the $l \times l$-minors of the matrix $D f$. On the level of the differential forms these equations are:

$$
d u=G_{\#} d^{\star} v, \quad d^{\star} u=d v=0 .
$$

In the light of these examples a search for linear PDEs in odd dimensions becomes quite appealing.

Question 1. Do there exist nonlinear forms with respect to $D f$ which solve linear partial differential equations?

Of particular interest would be to find such forms which are homogeneous of degree $\frac{n}{2}$ with respect to $D f$. The search for them is strongly motivated by the regularity problems (Liouville's Theorem, etc.) in quasiconformal analysis. Until very recently, every attempt had met with failure. Well, certain newly discovered conformations of the first order partials of the Möbius mappings in $\mathbb{R}^{n}$ turn out to satisfy the Laplace equation. In case $n=2 l$ these conformations are exactly the $l \times l$-minors of $D f$. It appears likely that within the next few years there will be much progress in elucidating what such quantities really are. Successively more refined attempts along these lines might eventually lead to a full set of the governing PDEs.

There is always further to go. Without getting into details we regard $u$ and $v$ as sections of the whole Grassmann algebra bundle $\wedge X=\oplus \wedge^{l} X$. Then there are elliptic Dirac operators $\bar{\partial}=d-d^{\star}$ and $\partial=d+d^{\star}$, defined on $W^{1, p}(\wedge X)$. Equations (7) consolidate into a single one:

$$
\bar{\partial}(u+v)=\mathcal{Q} \partial(u+v), \quad \text { with } \quad \mathcal{Q}=\frac{I-G_{\#}}{I+G_{\#}}: \wedge^{l} X \rightarrow \wedge^{l} X, \quad\|\mathcal{Q}\|_{L^{\infty}}<1 .
$$

This simple observation finds its generalization in the so-called Beltrami-Dirac equation:

$$
\bar{\partial} w=\mathcal{Q} \partial w \quad \text { for } w \in W^{1, p}(\wedge X),
$$

where $\mathcal{Q}: \wedge X \rightarrow \wedge X$ is now an arbitrary linear bundle map such that $\|\mathcal{Q}\|_{L^{\infty}}<1$.

In many respects this seems to be an excellent extension of the familiar complex Beltrami equation to all dimensions, though there is still room for variations of it [DS], [IM 1], [I 3]. The whole program is similar in spirit to Bojarski's work in the complex plane $[\mathrm{Bo}]$. Continuing this analogy we must first establish a firm foundation for the regularity theory of the Beltrami-Dirac equation. 
A principal feature of this new setting, unlike the nonlinear ones, is that the existence questions are fairly easy to deal with by the methods of elliptic PDEs. There seem to be, just as in $[\mathrm{As}]$ and $[\mathrm{EH}]$, certain fundamental patterns which in the end will yield qualitative $L^{p}$-estimates. It is quite relevant here to bring up the signature operator $S: L^{p}\left(\wedge^{l} X\right) \rightarrow L^{p}\left(\wedge^{l} X\right)$ which permutes $\partial$ and $\bar{\partial}$; in symbols $S \circ \partial=\bar{\partial}$, see [IM1] and $[\mathrm{LM}]$. This operator, also known as Beurling-Ahlfors transform, has been a source of new studies for many analysts, and much creative effort has gone into trying to compute the $p$-norms of $S$ [IM 2], [BL], [BW], [Li]. We will encounter related questions in Section 5.

To conclude this topic, let us take a look at some relations between the $l$-forms $d u$ and $d^{\star} v$ when $l$ is not half of the dimension. In this case $d u$ and $d^{\star} v$ are coupled nonlinearly:

$$
d^{\star} v=\mathcal{H}(d u)
$$

where $\mathcal{H}: \wedge^{l} X \rightarrow \wedge^{l} X$ is a given bundle map. Following the lead of the classical $p$ harmonic operator we notice that $\mathcal{H}$ is homogeneous of degree $p-1$ :

$$
\mathcal{H}(t \xi)=t^{p-1} \mathcal{H}(\xi), \quad \text { with } p=\frac{n}{l} .
$$

Here are more conditions:

$$
\begin{gathered}
|\mathcal{H}(\xi)-\mathcal{H}(\zeta)| \leq M|\xi-\zeta|(|\xi|+|\zeta|)^{p-2} \\
\langle\xi-\zeta, \mathcal{H}(\xi)-\mathcal{H}(\zeta)\rangle \geq m|\xi-\zeta|^{2}(|\xi|+|\zeta|)^{p-2}
\end{gathered}
$$

for all $\xi, \zeta \in \Gamma\left(\wedge^{l} X\right)$ and $t \geq 0$, where $0<m \leq M$ are constants. We refer to (9) as a Hodge system and to the pair $\left(d u, d^{\star} v\right) \in L^{p}\left(\wedge^{l} X\right) \times L^{q}\left(\wedge^{l} X\right), p+q=p q$, as $\mathcal{H}$-conjugate fields. Questing for further evidence of $\mathcal{H}$-conjugate fields leads to a nonlinear Hodgede Rham theory on manifolds with boundary [ISS], but it would take us a bit afield to present this theory here.

$\mathcal{H}$-conjugate fields can be uncoupled by applying the operators $d$ and $d^{\star}$ to the Hodge system. It results in the second order elliptic equations

$$
d^{\star} \mathcal{H}(d u)=0 \quad \text { and } \quad d \mathcal{H}^{-1}\left(d^{\star} v\right)=0 .
$$

They are certainly interesting from the PDEs and nonlinear potential theory perspective. However, such research no longer has as its goal to provide a solid underpinning for the quasiconformal theory. Regularity questions remain perfectly valid for the second order equations, in that they always lead to new estimates for quasiconformal mappings. But this seems to be a far less geometric route than the suggestions via the Beltrami-Dirac equation.

4. A quest for compactness. Many constructions in analysis and topology rely on limiting processes and the quasiconformal theory is no exception. At this point of development we do not yet have a well-defined list of compactness results for families of mappings with finite distortion. As an extreme example, it has been widely believed that the class of $K$-quasiconformal mappings in $\mathbb{R}^{n}$ is closed with respect to uniform convergence. This is not unexpected; it parallels precisely what happens in the complex plane. Unfortunately, most recent examples refuted this belief [I 4]. 
THEOREM 1. For each dimension $n \geq 3$ and dilatation $K>1$, there exists a sequence of quasiconformal mappings $f_{j}: \mathbb{R}^{n} \rightarrow \mathbb{R}^{n}, K\left(x, f_{j}\right) \equiv K$ for $j=1,2, \ldots$, converging uniformly to a linear map $f: \mathbb{R}^{n} \rightarrow \mathbb{R}^{n}$ whose dilatation is greater than $K$.

A cause of this anomaly is the failure of the rank-one-convexity of the linear distortion function in dimensions greater than 2. Let $\mathbb{R}^{n \times n}$ denote the vector space of real $n \times n$ matrices endowed with the norm $|A|=\max \{|A x| ;|x|=1\}$. A function $\mathcal{K}: U \rightarrow \mathbb{R}$, defined on an open set $U \subset \mathbb{R}^{n \times n}$, is said to be rank-one-convex at $A \in U$ if for every rank-one matrix $T \in \mathbb{R}^{n \times n}$ the real function $t \mapsto \mathcal{K}(A+t T)$ is convex near zero. In conformity with (1) the linear distortion function is defined on matrices with positive determinant by

$$
\mathcal{K}(A)=|A|\left|A^{-1}\right|=\frac{\max \{|A \xi| ;|\xi|=1\}}{\min \{|A \xi| ;|\xi|=1\}} .
$$

What Theorem 1 tells us, in particular, is that the standard Teichmüller metric approach to topology on the spaces of deformations for hyperbolic surfaces may have no counterpart in dimensions greater than two. One might ask:

QUESTION 2. What is the best possible estimate of the dilatation of the limit map?

At present [GI] the best known result is that

$$
K(f) \leq \lim _{j \rightarrow \infty}\left[\frac{K\left(f_{j}\right)+K^{n-1}\left(f_{j}\right)}{2}\right]^{\frac{2}{n}}
$$

and we conjecture that this estimate is best possible. Thus, in particular, we see that the dilatation of the limit map does not depend on the dimension, precisely we have

$$
K(f) \leq\left[\lim _{j \rightarrow \infty} K\left(f_{j}\right)\right]^{2},
$$

see also [V2] and [Ri]. This latter result might be central in the development of the infinite dimensional geometric function theory. There are alternatives galore:

TheOREM 2. Let $f_{j}: X \rightarrow Y$ be mappings of finite distortion, precisely it means that there are distortion functions $K_{j}: X \rightarrow[0, \infty)$ such that

$$
\left|D f_{j}(x)\right|^{n} \leq\left[K_{j}(x)\right]^{n-1} J\left(x, f_{j}\right) \quad \text { a.e. for } \quad j=1,2, \ldots
$$

Assume that $K_{j}$ are integrable and converge weakly to $K$ in $L^{1}(X)$, while $f_{j} \rightarrow f$ weakly in $W^{1, n}(X, Y)$. Then the above inequality remains valid for the limit map.

This result is new even in the classical case when the distortion functions are uniformly bounded, say $K_{j}(x)=K(x) \leq M$ for all $j=1,2, \ldots$ and $x \in X$. Critical for the proof was the observation that the matrix function, $A \mapsto\left(|A|^{n} / \operatorname{det} A\right)^{\frac{1}{n-1}}$, $\operatorname{det} A>0$, is polyconvex, that is, convex with respect to the variables $(\operatorname{det} A, A) \in \mathbb{R}_{+} \times \mathbb{R}^{n \times n}$. There are, of course, many more distortion functions of interest. The general question which we are facing is:

QUESTION 3. Which distortion functions are lower semicontinuous with respect to the weak convergence in $W^{1, n}(X, Y)$ ?

5. Sharp $L^{p}$-estimates versus variational integrals. As we said earlier, with reference to the Beurling-Ahlfors transform $S: L^{p}\left(\wedge^{l} \mathbb{R}^{n}\right) \rightarrow L^{p}\left(\wedge^{l} \mathbb{R}^{n}\right)$ the regularity 
theory [IM 1], [I 1], removable singularities [IMNS], distortion of Hausdorff dimension under quasiconformal mappings and so on, depend strongly on the p-norms of $S$. In dimension $n=2$ the utility of such estimates was already well understood due to the inequality

$$
\int_{\mathbb{C}}\left|f_{\bar{z}}\right|^{p} \leq A_{p}^{p} \int_{\mathbb{C}}\left|f_{z}\right|^{p}, \quad 1<p<\infty
$$

for complex functions $f \in W^{1, p}(\mathbb{C})$. A longstanding conjecture asserts that the smallest constant here is: $A_{p}=\max \left\{p-1, \frac{1}{p-1}\right\}$. It occurred to us that $A_{p}$ might very well be the norm of $S$ in all dimensions. In our attempt to answer this question, we have developed an effective method to secure dimension free estimates for some vector-valued Riesz transforms such as the signature operator $S: L^{p}\left(\wedge^{1} \mathbb{R}^{n}\right) \rightarrow L^{p}\left(\wedge^{1} \mathbb{R}^{n}\right)$ and many others [IM 2]. As a side benefit, we have identified the $p$-norms of the scalar Riesz transforms in $\mathbb{R}^{n}$. Other estimates, combined with the Fredholm index theory, have led us to new results for the linear elliptic PDEs with VMO-coefficients [IS 3]. But we cannot enter this topic here. In this section we shall take advantage of a new framework for the inequality (11) and similar ones in higher dimensions.

In a remarkable piece of work on sharp inequalities for martingales D. Burkholder [Bu] came up with a mysterious-looking inequality:

$$
(p-1)^{p-1}\left[(p-1)\left|f_{z}\right|-\left|f_{\bar{z}}\right|\right]\left(\left|f_{z}\right|+\left|f_{\bar{z}}\right|\right)^{p-1} \leq p^{p-2}\left[A_{p}^{p}\left|f_{z}\right|^{p}-\left|f_{\bar{z}}\right|^{p}\right], \quad p \geq 2 .
$$

What can one make of this? Well, the sharp constant in inequality (11) would follow from an affirmative answer to the following:

Question 4. For $f \in W^{1, p}(\mathbb{C})$ with $p \geq 2$, does the following inequality hold

$$
\int_{\mathbb{C}}\left[(p-1)\left|f_{z}\right|-\left|f_{\bar{z}}\right|\right]\left(\left|f_{z}\right|+\left|f_{\bar{z}}\right|\right)^{p-1} \geq 0 \quad ?
$$

Equality occurs here when for instance $f(z)=z \rho\left(|z|^{2}\right)$, where $\rho$ is a decreasing function of class $L^{p}(0, \infty)$ for which $t \mapsto t \rho(t)$ is increasing [BM-S]. As a matter of fact, these functions are among the stationary points for the corresponding Euler-Lagrange equation. The reason for preferring (13) to (11) is simply that the integrand, regarded as a function of the differential matrix $D f$, is rank-one-convex, which makes the Euler-Lagrage equation elliptic. We now recall an outstanding conjecture in the Calculus of Variations; for $n=2$ rank-one-convexity implies quasiconvexity (not true in higher dimensions [Š $]$ ). Follow Question 6 for a definition of quasiconvexity. This more general conjecture is likely to be easier to deal with, though it may seem very strange because, after all, it implies (13). It is fair to say, then, that Burkholder's approach to stochastic integrals is a perfectly valid procedure, in that it leads to probabilistically and geometrically significant problems.

There may be some elegant - and perhaps even simple - ways of characterizing the essence of inequality (13) in higher dimensions. To this end we begin with Hadamard's inequality $\operatorname{det} A \leq|A|^{n}$. Hence we see at once that $J(x, f)|D f(x)|^{p-n} \leq|D f(x)|^{p}$. On the average, however, we can do slightly better due to some internal cancellations. The 
cornerstone is the following estimate:

$$
\int_{\mathbb{R}^{n}} J(x, f)|D f(x)|^{p-n} d x \leq \lambda_{p}(n) \int_{\mathbb{R}^{n}}|D f(x)|^{p} d x
$$

for all $f \in W^{1, p}\left(\mathbb{R}^{n}, \mathbb{R}^{n}\right)$ with $p \geq n$, where $\lambda_{p}(n)$ is a positive constant less than 1 . This inequality is in all respects similar to (11) but its proof is not a straightforward consequence of the Calderón-Zygmund theory of singular integrals - far from it [IL1]. Another useful consequence of inequality (14) is a Caccioppoli type estimate for quasiregular mappings with exponents $p<n$. How far we can go below the dimension is of crucial importance for the size of removable singularities. That $\lambda_{p}(n) \geq 1$ for $p$ smaller than half of the dimension is clear from an example in [IM 1].

Question 5. Does inequality (14) hold with $\lambda_{p}(n)<1$ for all exponents $p>\frac{n}{2}$ ?

As yet, we have only handled the case $p>n-\varepsilon$ with some positive $\varepsilon=\varepsilon(n)$, by methods of nonlinear interpolation. The heart of our arguments is inequality (22); we put off discussing these methods to Section 7. Now, the natural direction to go would be to find the smallest constant $\lambda_{p}(n)$ for $p>n / 2$. We conjecture that $\lambda_{p}(n)=\left|1-\frac{n}{p}\right|$. This would give us a powerful base to work with sharp estimates for quasiregular mappings. Once again, we are faced with the question of rank-one-convexity of the integrand. The following result [I 6] will take us to an even larger context:

Theorem 3. The matrix function $\mathcal{F}: \mathbb{R}^{n \times n} \rightarrow \mathbb{R}$, defined by

$$
\mathcal{F}(A)=\left|1-\frac{n}{p}\right||A|^{p}-|A|^{p-n} \operatorname{det} A,
$$

is rank-one-convex for all $p \geq n / 2$. Furthermore, the factor $\left|1-\frac{n}{p}\right|$ is the smallest possible for this theorem to be true.

It is crucial to observe that $\mathcal{F}$ is homogeneous and rotationally invariant. If - and this is probably the case - it happens that under certain symmetries the rank-one-convexity implies quasiconvexity then (14) would hold with $\lambda_{p}(n)=\left|1-\frac{n}{p}\right|$, as desired. Could one devise a much fancier method?

QUESTION 6. Is the function $\mathcal{F}: \mathbb{R}^{n \times n} \rightarrow \mathbb{R}$ quasiconvex?

Recall that $\mathcal{F}$ is quasiconvex if

$$
\int[\mathcal{F}(A+D h)-\mathcal{F}(A)] \geq 0
$$

for every constant matrix $A \in \mathbb{R}^{n \times n}$ and all smooth mappings $h: \mathbb{R}^{n} \rightarrow \mathbb{R}^{n}$ with compact support. As in (13), equality occurs in a number of interesting cases [I 6].

Entering into variational problems, I would like to mention my work with A. Lutoborski [IL 1,2] in which some specific nonconvex variational integrals have been analyzed in great detail, see also [AD]. These efforts resulted in proving the existence of the minima of certain energy functionals of the form $\mathcal{E}[f]=\int_{\Omega} E(D f)$. Here the integrand $E: \mathbb{R}^{n \times n} \rightarrow \mathbb{R}_{+}$vanishes exactly on the matrices of conformal transformations (potential wells). In that sense the energy $\mathcal{E}[f]$ measures how far a given deformation $f: \Omega \rightarrow \mathbb{R}^{n}$ is from a conformal one. The minimizers are called nearly conformal mappings. Variational 
integrals, whose potential wells consist of conformal deformations, might be of some interest in both quasiconformal theory and nonlinear elasticity as they provide effective means of extending a given boundary map to a nearly conformal one. Perhaps the most instructive example of all is:

$$
\mathcal{E}_{p}[f]=\int_{\Omega}\left[|D f(x)|^{n}-J(x, f)\right]^{p} d x, \quad 1 \leq p<\infty .
$$

The integrand $E(A)=\left(|A|^{n}-\operatorname{det} A\right)^{p}$, being a convex function of the variables $(\operatorname{det} A, A)$ $\in \mathbb{R} \times \mathbb{R}^{n \times n}$ is polyconvex. It fails to be coercive, however. The lack of coercivity is compensated by a rather nontrivial estimate, referred to as mean coercivity:

$$
\delta \int_{\Omega}|D f|^{n p} \leq \mathcal{E}_{p}[f]+\int_{\Omega}|D g|^{n p}, \quad \delta=\delta(n, p)>0 .
$$

Here $g \in W^{1, n p}\left(\Omega, \mathbb{R}^{n}\right)$ represents the given Dirichlet data while $f$ is the unknown deformation subject to the boundary condition $f-g \in W_{0}^{1, n p}\left(\Omega, \mathbb{R}^{n}\right)$. Having this estimate we implemented the direct method of the calculus of variations [Da] to show that the functional $\mathcal{E}_{p}$ indeed attains its minimum. A puzzling thing about this variational problem is the question of uniqueness; there are enough arguments to safely conjecture that $\mathcal{E}_{p}$ admits only one minimizer. However, the failure of monotonicity of the derivative map $E^{\prime}: \mathbb{R}^{n \times n} \rightarrow \mathbb{R}^{n \times n}$ stands in the way. The following weaker problem having a good chance of being solved, would come to the rescue.

Question 7. Is the mapping $E^{\prime}$ monotone in the average sense?

Precisely we expect that

$$
\int_{\Omega}\left\langle E^{\prime}(D f)-E^{\prime}(D g) \mid D f-D g\right\rangle \geq \delta \int_{\Omega}|D f-D g|^{2}(|D f|+|D g|)^{n p-2}
$$

whenever $f-g \in W_{0}^{1, n p}\left(\Omega, \mathbb{R}^{n}\right)$, where $\delta=\delta(n, p)>0$.

While it has not been possible to provide definite answers to the uniqueness problem for general polyconvex functionals the recent a priori estimates for null Lagrangians [IL 1] and nonlinear commutators [I 2] seem to work in specific cases.

6. Very weak solutions. Related and perhaps most intriguing is the question of uniqueness for the so-called $\mathcal{H}$-harmonic equation $d^{\star} \mathcal{H}(d u)=d^{\star} f$. Recall conditions (10) for the bundle map $\mathcal{H}: \wedge^{l} X \rightarrow \wedge^{l} X$. For a given $l$-form $f \in L^{q}\left(\wedge^{l} X\right), \frac{1}{p}+\frac{1}{q}=1$, we seek solution $u \in W^{1, p}\left(\wedge^{l-1} X\right)$ with vanishing tangential component on $\partial X$. This is what we call the natural setting of the $\mathcal{H}$-harmonic problem. Browder's theory of monotone operators ensures the existence and uniqueness of such solutions [ISS], [Sc]. This in turn gives rise to a nonlinear operator $\mathbb{H}: L^{q}\left(\wedge^{l} X\right) \rightarrow L^{p}\left(\wedge^{l} X\right)$ which carries given element $f \in L^{q}\left(\wedge^{l} X\right)$ into $d u \in L^{p}\left(\wedge^{l} X\right)$. Of course, the space $L^{q}\left(\wedge^{l} X\right)$ is a natural domain of $\mathbb{H}$. But it is also of great interest to know whether $\mathbb{H}$ extends as a continuous operator to other Lebesgue spaces. The following exact request sounds plausible

QUESTION 8. What is the range of the parameter $\lambda$ for which we have the inequality

$$
\|\mathbb{H} f-\mathbb{H} g\|_{\lambda p}^{p-1} \leq C_{p}\|f-g\|_{\lambda q}^{\alpha}\left(\|f\|_{\lambda q}+\|g\|_{\lambda q}\right)^{1-\alpha}
$$

with $f, g \in L^{\lambda q}\left(\wedge^{l} X\right)$ and some exponent $0<\alpha=\alpha(p, \lambda) \leq 1$ ? 
This is undoubtedly true if $\lambda=1$, but the estimate beyond the natural exponents $(\lambda \neq 1)$ is likely to be far harder; it remains unsolved even for the $p$-harmonic equation $\operatorname{div}|\nabla u|^{p-2} \nabla u=\operatorname{div} f$. What is known, however, is that $\lambda$ can be any number in some closed interval $[a, b]$, where $\max \left\{\frac{1}{p}, \frac{1}{q}\right\} \leq a<1<b$. We should also mention that the case $g \equiv 0$ has been settled and developed much further [I 1], [IS 2], but our lectures are too short to contain it. Note that this latter case handles inequality (19) when $\mathcal{H}$ is a linear map. For compact manifolds and $\lambda>1$ we do not even need inequality (19); the uniqueness follows from the embedding $L^{\lambda p}\left(\wedge^{l} X\right) \subset L^{p}\left(\wedge^{l} X\right)$. It is the case of the very weak solutions $(\lambda<1)$ that drives us into truly new investigations concerning nonlinear commutators. We will discuss this a little further on. Although it strays somewhat from the focus of our lectures, let me mention two relevant results only recently established. But first, we need a definition:

Definition 2. The "grand" Lebesgue space $L^{s)}\left(\wedge^{l} X\right), 1<s<\infty$, consists of $l$-forms

$$
\varphi \in \bigcap_{1 \leq r<s} L^{r}\left(\wedge^{l} X\right)
$$

such that the expression $(s-r)\|\varphi\|_{r}^{r}$ stays bounded as $r$ increases to $s$. This space is furnished with the norm

$$
\|\varphi\|_{s)}=\sup \left\{\sqrt[s]{\varepsilon}\|\varphi\|_{s-\varepsilon} ; 0<\varepsilon \leq s-1\right\} .
$$

It then follows that the closure of $L^{s}\left(\wedge^{l} X\right)$, denoted by $\mathcal{L}^{s}\left(\wedge^{l} X\right)$, is made up of forms such that $\lim _{\varepsilon \downarrow 0} \varepsilon \int|\varphi|^{s-\varepsilon}=0$. In a manner consistent with the above, we introduce the "grand" Sobolev spaces $W^{1, s)}\left(\wedge^{l} X\right)$ and $\mathcal{W}^{1, s)}\left(\wedge^{l} X\right)$.

In [GIS] we succeeded in proving inequality (19) for $f, g \in L^{q)}\left(\wedge^{l} X\right)$, where obviously the subscripts $\lambda p$ and $\lambda q$ must be replaced by $p$ ) and $q$ ), respectively. These peculiar spaces occur in various contexts [IS1], [G], [GISS]. Further, our new results in [GIS] put more life into them, for example:

ThEOREM 4. For each finite signed measure $\mu$ on $\Omega \subset \mathbb{R}^{n}$ the $n$-harmonic equation

$$
\operatorname{div}|\nabla u|^{n-2} \nabla u=\mu
$$

has a unique solution $u \in W_{0}^{1, n)}(\Omega)$.

As far as we are aware this is the first time a uniqueness question for the very weak solutions has been solved for nonlinear PDEs. In addition, uniform estimates reveal that:

THEOREM 5. If $|\mu| \ll d x$, then $u \in \mathcal{W}_{0}^{1, n)}(\Omega)$. This simply means that $u \in W_{0}^{1, p}(\Omega)$ for every $1 \leq p<n$ and

$$
\lim _{\varepsilon \downarrow 0} \varepsilon \int|\nabla u|^{n-\varepsilon}=0 .
$$

For related questions concerning PDEs with measure in the right hand side see [BG], [DHM 1,2], [Mu2] and [KM].

7. Nonlinear commutators. There is one recurring idea in our methods which we have not yet mentioned, and that is the phenomenon of cancellation observed in vari- 
ous nonlinear differential and integral expressions. Before embarking on that discussion, however, we need to recall some nonlinear commutators [I 2].

Let $(X, \mu)$ be a measure space and let $T: L^{p}(X, \mathbb{C}) \rightarrow L^{p}(X, \mathbb{C})$ be a bounded linear operator with $p \in\left[p_{1}, p_{2}\right]$ and $1 \leq p_{1}<p_{2} \leq \infty$. The object of our discussion here is the commutator of $T$ and the power function $f \mapsto|f|^{z} f$, that is:

$$
T^{z} f=T\left(|f|^{z} f\right)-|T f|^{z} f, \text { where } \frac{p_{1}}{p}-1 \leq \operatorname{Re} z \leq \frac{p_{2}}{p}-1 .
$$

In order to get quickly to the substance of the problems we formulate, as a starting point, the most general and precise estimate:

$$
\left\|T^{z} f-T^{z} h\right\|_{\frac{p}{a}} \leq C_{p} \frac{|z|}{r}\|f-h\|_{p}^{a-r a}\left(\|f\|_{p}^{a r}+\|h\|_{p}^{a r}\right)
$$

for $|z| \leq r$. Here $a=1+\operatorname{Re} z$ and $r$ is sufficiently small.

The proof makes use of the complex method of interpolation. Note that the function $z \mapsto T^{z} f$ is analytic in $z$. Taking the derivative at zero we obtain what is known as the Rochberg-Weiss commutator

$$
T^{\log } f=T(f \log |f|)-(T f) \log |T f| .
$$

Next, passing to the limit in (20) yields

$$
\left\|T^{\log } f-T^{\log } h\right\|_{p} \leq C_{p}\|f-h\|_{p} \log \left(e+\frac{\|f\|_{p}+\|h\|_{p}}{\|f-h\|_{p}}\right)
$$

In particular,

$$
\|T(f \log |f|)-(T f) \log |T f|\|_{p} \leq C_{p}\|f\|_{p} .
$$

We emphasize that the individual terms here need not belong to $L^{p}(X, \mathbb{C})$, but the difference does. This cancellation effect was first observed by R. Rochberg and G. Weiss in their study of analytic families of Banach spaces [RW]. There has since been more systematic work done by N. J. Kalton [K1, 2], R. Rochberg [Ro] and M. Milman [Mi], $[\mathrm{MR}]$, and many others $[\mathrm{CJMR}],[\mathrm{MS}],[\mathrm{RW}]$. As a matter of fact, in our first attempt to understand the very weak solutions of PDEs [I 1], we have already established the following inequality:

$$
\left\|T\left(f|f|^{-\varepsilon}\right)\right\|_{\frac{p}{1-\varepsilon}} \leq C_{p}|\varepsilon|\|f\|_{p}^{1-\varepsilon}, \quad \text { for } f \in L^{p}\left(\mathbb{R}^{n}\right) \cap \operatorname{ker} T .
$$

The proof did not depend on the commutators but worked only for a certain type of singular integral operators $T$, sufficient for applications. Of course, the significance of this inequality is due to the factor $|\varepsilon|$ in the right hand side. Seemingly obvious improvement in (20) over (22) turns out to be extremely powerful in new applications to PDEs and the theory of the Jacobians. Take, for instance, Theorem 7 in the last section.

An elementary device of averaging inequality (20) with respect to the complex parameter $z$ leads to new commutators whose natural domains are Orlicz spaces. Without getting into technicalities, for a given function $\mathcal{A}$ one might consider the commutator $T^{\mathcal{A}}=T \mathcal{A}-\mathcal{A} T: L^{N}(X) \rightarrow L^{M}(X)$, where $N, M: \mathbb{R}_{+} \rightarrow \mathbb{R}_{+}$are the Young functions defining the norms \|\|$_{N}$ and \|\|$_{M}$ in the corresponding Orlicz spaces. The natural generalization of the Rochberg-Weiss inequality reads as follows:

$$
\left\|T^{\mathcal{A}} f\right\|_{M}=\|T \mathcal{A}(f)-\mathcal{A}(T f)\|_{M} \leq C\left(\|f\|_{N}\right) \quad \text { for } f \in L^{N}(X) .
$$


For this inequality to be true, we must assume certain relations between the Young functions and the given function $\mathcal{A}$. Because of lack of homogeneity, $C$ is no longer a constant here but a function on $\mathbb{R}_{+}$. Only in the case of $\mathcal{A}(t)=t \log |t|$, it stands for the multiplication by a constant. Let us introduce the so-called indicator $I(t)=$ $\mathcal{A}(t) / M^{-1}(N(t))$, which tells us how much degree of integrability we gain due to the (well hidden) cancellation in (23). It appears that we gain nothing if $\mathcal{A}$ is too far from the linear function, say $\mathcal{A}(t) \approx t^{a}$ with $a \neq 1 ; I(t)$ is simply bounded at infinity. Nevertheless, in this case an internal cancellation effect still takes place, as we gain the presence of $\varepsilon$ on the right hand side of inequality $(22)$.

Here are two partial results: For $\mathcal{A}_{\alpha}(t)=t \log ^{\alpha}(e+t)$ and $\alpha \in(0,1]$ inequality (23) holds with $N_{\alpha}(t)=t^{p} \log ^{\alpha-1}(e+t)$ and $M_{\alpha}(t)=t^{p} \log ^{(1-\alpha)(p-1)}(e+t)$, where the indicator equals $I(t)=\log (e+t)$, for each $\alpha \in(0,1]$ and $1<p<\infty$. In order to gain more cancellation we must take $\mathcal{A}$ closer to the identity function. We choose as an illustration: $\mathcal{A}(t)=\frac{d}{d \alpha} \mathcal{A}_{\alpha}(t)=t \log \log (e+t)$ at $\alpha=0$. Then (23) remains valid with the pair of Young functions $N_{0}$ and $M_{0}$, in which case we have $I(t)=\log (e+t) \log \log (e+t)$. All the above results are qualitatively optimal and amount to the principle:

The further $\mathcal{A}$ is from the identity the less cancellation occurs in the commutator.

8. Jacobians and wedge products. The effect of cancellation in the nonlinear commutators plays an important role in the study of the Jacobians and compensated compactness. We shall report on it briefly; the interested reader may wish to consult my notes of Lipschitz Lectures [I 2]. The primary goal of these numerous lectures was to bridge a gap between the nonlinear interpolation and the actual use and interpretation of the results in a variety of disciplines in classical analysis, see also [I 5] for an extended survey.

Let $f=\left(f^{1}, \ldots, f^{n}\right): X \rightarrow \mathbb{R}^{n}$ be a mapping in Sobolev space $W^{1, n}\left(X, \mathbb{R}^{n}\right)$, thus its Jacobian determinant induces an $n$-form on $X$. Precisely we have

$$
J(x, f) d x=d f^{1} \wedge \ldots \wedge d f^{n},
$$

the pullback of $d y=d y_{1} \wedge \ldots \wedge d y_{n}$. Some special properties of the Jacobian have already been appreciated in the late 1960's when F. W. Gehring [Ge], J. Väisälä [V 1] and Y. Reshetnyak [Re 1] laid down the foundation of quasiconformal mappings. It is important to recognize, in this connection, the remarkable contribution of H. Wente [W] as well. Modern language better suited for dealing with the Jacobians is that of the exterior algebra. The Jacobian can be viewed as a wedge product $J(x, f) d x=\varphi^{1} \wedge \ldots \wedge \varphi^{m}$, where each factor $\varphi$ takes the form $\varphi=d f^{i_{1}} \wedge \ldots \wedge d f^{i_{l}}$. This is the view which we shall adopt and extend suitably.

Let $\mathbf{p}=\left(p_{1}, \ldots, p_{m}\right), 1<p_{1}, \ldots, p_{m}<\infty$, be a Hölder conjugate sequence, that is $\frac{1}{p_{1}}+\ldots+\frac{1}{p_{m}}=1$, and let $\mathbf{l}=\left(l_{1}, \ldots, l_{m}\right)$ be an $m$-tuple of positive integers with $l_{1}+\ldots+l_{m}=n$. We let $L^{\mathbf{p}}\left(\wedge^{1} X\right)$ denote the space of $m$-tuples $\Phi=\left(\varphi^{1}, \ldots, \varphi^{m}\right)$ of differential forms $\varphi^{i} \in L^{p_{i}}\left(\wedge^{l_{i}} X\right)$ and continue to write $\operatorname{det} \Phi=*\left(\varphi^{1} \wedge \ldots \wedge \varphi^{m}\right) \in L^{1}(X)$. This more general setting unifies earlier work in many other types of nonlinear differential situations. For example, as shown in [RRT] and [IL 1], the compensated compactness of F. Murat [Mu1] and L. Tartar [Ta] is in fact a statement on weak continuity of the 
wedge products of differential forms under suitable integrability assumptions on $d \Phi=$ $\left(d \varphi^{1}, \ldots, d \varphi^{n}\right)$.

Let us begin with a simple estimate which is straightforward from Hölder's inequality:

$$
\left|\int_{X} \eta(\operatorname{det} \Phi-\operatorname{det} \Psi)\right| \leq C_{\mathbf{p}}\|\eta\|_{\infty} \sum_{k=1}^{m}\left\|\varphi^{k}-\psi^{k}\right\|_{p_{k}} \prod_{i \neq k}\left\|\left|\varphi^{i}\right|+\left|\psi^{i}\right|\right\|_{p_{i}}
$$

for each test function $\eta \in C_{0}^{\infty}(X)$. Hence, in particular, det $: L^{\mathbf{p}}\left(\wedge^{\mathbf{l}} X\right) \rightarrow L^{1}(X)$ is a continuous operator. In order to obtain something deeper than that we must confine ourselves to closed forms and combine $L^{p}$-Hodge theory with commutator results. For the sake of clarity, think of $X$ as $\mathbb{R}^{n}$.

First, one can replace the supremum norm of the test function by its BMO-norm. Thus (24) holds for $\eta \in V M O$ as well. By $\mathcal{H}^{1}$-BMO duality we then conclude that $\operatorname{det} \Phi$ actually belongs to the Hardy space $\mathcal{H}_{l o c}^{1}(X)$. This elegant result was first established in $\mathbb{R}^{n}$ by R. Coifman, P. Lions, Y. Meyers and S. Semmes in 1989. We refer to [CLMS], [CG], [EM], [IV 1] and [Se] for some generalizations. In addition, as a side benefit of (24), we find that the operator $\operatorname{det}: L^{\mathbf{p}}\left(\wedge^{\mathbf{l}} X\right) \rightarrow \mathcal{H}_{\text {loc }}^{1}(X)$ is not only bounded but also continuous [I 2].

A precise estimate for $X=\mathbb{R}^{n}$ reads as follows:

$$
\|\operatorname{det} \Phi-\operatorname{det} \Psi\|_{\mathcal{H}^{1}} \leq C_{\mathbf{p}} \sum_{k=1}^{m}\left\|\varphi^{k}-\psi^{k}\right\|_{p_{k}} \prod_{i \neq k}\left\|\left|\varphi^{i}\right|+\mid \psi^{i}\right\|_{p_{i}}
$$

for $\Phi, \Psi \in L^{\mathbf{P}}\left(\wedge^{\mathbf{l}} \mathbb{R}^{n}\right) \cap$ ker $d$. This in case of mappings $f, g \in W^{1, n}\left(\mathbb{R}^{n}, \mathbb{R}^{n}\right)$ reduces to

$$
\|\mathcal{J}(, f)-\mathcal{J}(, g)\|_{\mathcal{H}^{1}} \leq C(n)\|D f-D g\|_{n}\left(\|D f\|_{n}+\|D g\|_{n}\right)^{n-1} .
$$

Integration by parts and Sobolev embedding theorem give improvements in other directions. For instance, inequality (24) remains valid if we replace the norms \|\|$_{p_{i}}$ by \|\|$_{s_{i}}$ and $\|\eta\|_{\infty}$ by $\|\nabla \eta\|_{\infty}$, where $\mathbf{s}=\left(s_{1}, \ldots, s_{m}\right)$ is only assumed to be a Sobolev conjugate sequence, that is $\frac{1}{s_{1}}+\ldots+\frac{1}{s_{m}}=1+\frac{1}{n}$ and $1 \leq s_{1}, \ldots, s_{m} \leq \infty$. Although the $p$-norms of $\Phi$ and $\Psi$ do not enter into the improved estimate it is still required that the forms $\Phi$ and $\Psi$ are in $L^{\mathbf{P}}\left(\wedge^{1} X\right)$. Nevertheless, by an approximation, we can get rid of this requirement and give meaning to $\operatorname{det} \Phi$ as a Schwartz distribution of order one for all forms $\Phi$ of class $L^{\mathbf{s}}\left(\wedge^{\mathbf{l}} X\right) \cap \operatorname{ker} d$. This is what we call the weak wedge product or weak Jacobian. The value of the distribution $\operatorname{det} \Phi$ on a test function $\eta \in C_{0}^{\infty}(X)$ can be defined as

$$
(\eta, \operatorname{det} \Phi)=\lim _{j \rightarrow \infty} \int_{X} \eta \operatorname{det} \Phi_{j}
$$

where $\Phi_{j}$ are in $L^{\mathbf{p}}\left(\wedge^{\mathbf{l}} X\right) \cap$ ker $d$ and converge to $\Phi$ in $L^{\mathbf{s}}\left(\wedge^{\mathbf{l}} X\right)$. That such an approximation of $\Phi$ exists requires some work.

More radical attempts lead to many other weakly defined nonlinear forms. To illustrate, we consider the operator $(\operatorname{det} D) \log |D|$ which carries a given mapping $f: \mathbb{R}^{n} \rightarrow \mathbb{R}^{n}$ into $(\operatorname{det} D f) \log |D f|$. This operator turns out to be a continuous map from $W^{1, n}\left(\mathbb{R}^{n}, \mathbb{R}^{n}\right)$ into $\mathcal{D}^{\prime}\left(\mathbb{R}^{n}\right)$. A vehicle for defining (det $\left.D f\right) \log |D f|$ as a Schwartz distribution is the nonlinear commutator $T^{i t} h=T\left(|h|^{i t} h\right)-|T h|^{i t} T h$. Estimate (20) reads as

$$
\left\|T^{i t} f-T^{i t} h\right\|_{p} \leq C_{p}|t|\|f-h\|_{p}^{1-\alpha}\left(\|f\|_{p}^{\alpha}+\|h\|_{p}^{\alpha}\right)
$$


with some $0<\alpha<1$. The latter inequality ensures the existence of the derivative $i \frac{d}{d t}\left(|D f|^{i t} \operatorname{det} D f\right)$ at $t=0$, and this derivative is one of the possible interpretations of (det $D f) \log |D f|$. We emphasize that the difference quotients defining the derivative converge only in $\mathcal{D}^{\prime}\left(\mathbb{R}^{n}\right)$. A slightly more general result, which highlights the role of the $\mathcal{H}^{1}$-BMO duality, seems to be very elegant. Inspired by this, we [IV 2] were studying the nonlinear map $\mathcal{Z}: \mathcal{H}^{1}\left(\mathbb{R}^{n}\right) \rightarrow \mathcal{D}^{\prime}\left(\mathbb{R}^{n}\right)$ which takes a given element $h$ from the Hardy space to the function $\mathcal{Z} h=h \log \left(e+\frac{|h|}{\|h\|_{1}}\right)$. Although this function need not be locally integrable we were able to give a meaning to $\mathcal{Z} h$ as a Schwartz distribution of order 1. Worth mentioning is that the statement $\mathcal{Z} h \in L^{1}\left(\mathbb{R}^{n}\right)$ amounts to saying that $h$ belongs to the Zygmund space $L \log L\left(\mathbb{R}^{n}\right)$, and also $\|\mathcal{Z} h\|_{1}$ defines a norm in this space. The triangle inequality remains well short of clear.

For a distribution $T \in \mathcal{D}^{\prime}\left(\mathbb{R}^{n}\right)$ of order 1 we define seminorms $[T]_{R}=\sup \{|T \varphi|$; $\left.\varphi \in C_{0}^{\infty}(\mathbb{B}),\|\nabla \varphi\|_{\infty}=1\right\}$, the supremum being taken over all balls $\mathbb{B} \subset \mathbb{R}^{n}$ of radius $R$. Our main estimate in [IV 1] reads as:

$$
[\mathcal{Z} g-\mathcal{Z} h]_{R} \leq C_{R}(n)\|g-h\|_{\mathcal{H}^{1}} \log \left(e+\frac{\|g\|_{\mathcal{H}^{1}}+\|h\|_{\mathcal{H}^{1}}}{\|g-h\|_{\mathcal{H}^{1}}}\right)
$$

Recall a result of E. Stein [S]: if $h \in \mathcal{H}^{1}\left(\mathbb{R}^{n}\right)$ and $h \geq 0$ in an open set $\Omega \subset \mathbb{R}^{n}$, then $h \log h \in L_{\text {loc }}^{1}(\Omega) \subset \mathcal{D}^{\prime}(\Omega)$. Ultimately, inequality (25) reveals an interesting fact that deserves a statement of its own:

THEOREM 6. If $h_{k} \rightarrow h$ in $\mathcal{H}^{1}\left(\mathbb{R}^{n}\right)$ and $h_{k} \geq 0$ on an open region $\Omega \subset \mathbb{R}^{n}$, then $h_{k} \rightarrow h$ in $L \log L(E)$ on every compact $E \subset \Omega$.

The point is that $h_{k}-h$ may change sign in $\Omega$. But this is another subject, and we will not go into it here. Instead, we conclude with one corollary which complements the familiar result of S. Müller [Mü]:

COROLlaRY 1. Suppose that the orientation preserving mappings $f_{k}: \Omega \rightarrow \mathbb{R}^{n}$ converge to $f$ in $W^{1, n}\left(\Omega, \mathbb{R}^{n}\right)$. Then $\operatorname{det} D f_{k} \rightarrow \operatorname{det} D f$ in $L \log L(E)$ for each compact $E \subset \Omega$.

Continuing in this fashion, one can try a setting of greater generality. In [GIM], we have identified those pairs $N, \mathcal{L}$ of Orlicz functions for which the expression $\mathcal{L}(|D f|) \operatorname{det} D f$ is locally integrable on $\Omega \subset \mathbb{R}^{n}$, whenever $f: \Omega \rightarrow \mathbb{R}^{n}$ is orientation preserving and belongs to the Orlicz-Sobolev space $W^{1, N}\left(\Omega, \mathbb{R}^{n}\right)$. Now, given an arbitrary mapping $f \in W^{1, N}\left(\Omega, \mathbb{R}^{n}\right)$ (not necessarily orientation preserving) it is natural to interpret the expression $\mathcal{L}(|D f|) \operatorname{det} D f$ as a Schwartz distribution.

QUESTION 9. Is the operator $\mathcal{L}(|D|) \operatorname{det} D: W^{1, N}\left(\Omega, \mathbb{R}^{n}\right) \rightarrow \mathcal{D}^{\prime}(\Omega)$ continuous?

For orientation preserving mappings in $W^{1, N}\left(\Omega, \mathbb{R}^{n}\right)$, one might try to generalize Corollary 1 accordingly.

In drawing this section to a close, we shall address one more question about the Jacobian operator $\mathcal{J}: W^{1, n p}\left(\mathbb{R}^{n}, \mathbb{R}^{n}\right) \rightarrow \mathcal{H}^{p}\left(\mathbb{R}^{n}\right), \mathcal{J} f=\operatorname{det} D f$, where $\mathcal{H}^{p}\left(\mathbb{R}^{n}\right)$ stands for the usual Lebesgue space $L^{p}\left(\mathbb{R}^{n}\right)$ if $p>1$. A continuous map

$$
\mathcal{E}: \mathcal{H}^{p}\left(\mathbb{R}^{n}\right) \rightarrow W^{1, n p}\left(\mathbb{R}^{n}, \mathbb{R}^{n}\right)
$$

is said to be a fundamental solution for $\mathcal{J}$ if $\mathcal{J} \circ \mathcal{E}=I d: \mathcal{H}^{p}\left(\mathbb{R}^{n}\right) \rightarrow \mathcal{H}^{p}\left(\mathbb{R}^{n}\right)$. 
QUESTION 10. Does there exist a fundamental solution for the Jacobian operator?

If so, we could solve in a canonical way the Jacobian equation:

$$
\operatorname{det} D f(x)=\lambda(x) \quad \text { so that } \quad\|D f\|_{n p}^{n} \leq C(n, p)\|\lambda\|_{\mathcal{H}^{p}\left(\mathbb{R}^{n}\right)}
$$

for $f \in W^{1, n p}\left(\mathbb{R}^{n}, \mathbb{R}^{n}\right)$ with any given $\lambda \in \mathcal{H}^{p}\left(\mathbb{R}^{n}\right)$. Solving (26) is an invitation to absolutely new studies in the theory of genuine nonlinear PDEs. Implications to the theory of the Monge-Ampère equation would be undoubtedly new and valuable. Following $[\mathrm{DM}]$ we can show that the Jacobian equation admits solutions for $\lambda \in C_{0}^{\infty}\left(\mathbb{R}^{n}\right)$ with integral zero. However, these solutions do not necessarily satisfy the uniform bound in (26). In this way we are naturally led to a minimization problem for the convex variational integral $I[f]=\int_{\mathbb{R}^{n}}|D f|^{n p}$ subject to the volume constraint $\operatorname{det} D f(x)=\lambda(x)$, a.e. It is not difficult to see that the minimum is actually attained. It may very well be that the minimizer is unique (up to rotations) and, as such, can be used to define the fundamental solution for the Jacobian equation. For the related questions see $[\mathrm{Ba}],[\mathrm{BK}],[\mathrm{M}],[\mathrm{McM}]$, $[\mathrm{R}],[\mathrm{RY}]$ and $[\mathrm{Ye}]$.

9. Degree formulas. In a very pragmatic sense, the integral $\int_{X} \mathcal{J}(x, f) d x$ encodes some topological invariants of the mapping $f: X \rightarrow Y$, including its degree; the number of times $Y$ is covered by $f(X)$. A clear trend has emerged in nonlinear elasticity and geometric PDEs such as Ginzburg-Landau equation, harmonic maps, and so on $[\mathrm{BBH}]$, $[\mathrm{EsM}],[\mathrm{GMS}]$ : the trend to figure out if and how it is possible to define the degree of maps with nonintegrable Jacobian. A fundamental character of our estimates can now be attested by applications to this problem. Before we jump to the conclusions, though, let us acquaint ourselves with the estimates concerning power type perturbations of the determinant, say $\varphi^{1} \wedge \ldots \wedge \varphi^{m}\left(\left|\varphi^{1}\right| \ldots\left|\varphi^{m}\right|\right)^{-\varepsilon}$. Here we assume that the form $\Phi=$ $\left(\varphi^{1}, \ldots, \varphi^{m}\right)$ is closed and belongs to the grand space $\left.L^{\mathbf{p}}\right)\left(\wedge^{\mathbf{l}} X\right)$, see Definition 2 . Using inequality (22) and the Hodge-de Rham decomposition we can approximate $\left|\varphi_{i}\right|^{-\varepsilon} \varphi_{i}$ by closed forms with enough accuracy to conclude that

$$
\varlimsup_{\varepsilon \downarrow 0} \int_{X} \eta \operatorname{det} \Phi|\operatorname{det} \Phi|^{-\varepsilon}<\infty
$$

for every $\eta \in C_{0}^{\infty}(X)$. Not only that, but the limit actually exists if $\Phi \in \mathcal{L}^{\mathbf{p}}\left(\wedge^{\mathbf{l}} X\right) \cap \operatorname{ker} d$, see Definition 2. In general, the determinant need not be integrable and we cannot pass to the limit in the integrand. What interests us most is the case when $X$ is a compact $n$-manifold without boundary so we can take $\eta=1$ and quote the limit in (27) as "weak integral" of the determinant. This suggests the possibility of extending the notion of the degree to mappings with weakly integrable Jacobians. For a recent account of the degree theory along more classical lines we refer to H. Brezis and L. Nirenberg [BN1]. In that paper the authors define and establish basic properties of the degree of VMO-maps $f: X \rightarrow Y$ between $n$-dimensional compact (without boundary) Riemannian manifolds $X$ and $Y$. Notice that $V M O(X, Y)$ contains the Sobolev space $W^{1, n}(X, Y)$; the latter presents no difficulty because $C^{\infty}(X, Y)$ is dense in $W^{1, n}(X, Y)$ and Jacobians are $L^{1}$ integrable. We soon realized [GISS] that (27) opens a new route to the degree of mappings in some Orlicz-Sobolev classes weaker than $W^{1, n}(X, Y)$. 
In our method the $l$-th cohomology group $\mathcal{H}^{l}(Y)$ of the target manifold is required to be nontrivial for at least one integer $1<l<n$, which unfortunately excludes the spheres. The trouble is that if $\mathcal{H}^{l}(Y)=0$ there do not exist closed forms $\alpha \in C^{\infty}\left(\wedge^{l} Y\right)$ and $\beta \in C^{\infty}\left(\wedge^{n-l} Y\right)$ such that $\int_{Y} \alpha \wedge \beta=1$. Indeed, $\alpha$ as a closed $l$-form is exact, so is the $n$-form $\omega=\alpha \wedge \beta$ and thus $\int_{Y} \omega=0$. On the other hand, if we can find a harmonic field $\theta \in \mathcal{H}^{l}(Y)$ with $\int_{Y}|\theta|^{2}=1$, then $\omega=\theta \wedge * \theta$ does the job. This is just another way of seeing that $\mathcal{H}^{l}(Y)$ must be nontrivial.

Now, with the assumptions above, we define the Jacobian of $f: X \rightarrow Y$ by simply pulling back the $n$-form $\omega$ via $f$. Precisely, we have: $\mathcal{J}(x, f) d x=f^{\#} \omega=f^{\#} \alpha \wedge f^{\#} \beta=$ $\varphi^{1} \wedge \varphi^{2}$. It is very crucial to understand that $\varphi^{1}$ and $\varphi^{2}$ are closed forms only when $f$ is sufficiently regular. Later on, we dispense with this requirement by an approximation. By virtue of (27) it is tempting to introduce the degree of $f$ by

$$
\operatorname{deg}(f ; X, Y)=\lim _{\varepsilon \downarrow 0} \int_{X} \frac{\mathcal{J}(x, f) d x}{|\mathcal{J}(x, f)|^{\varepsilon}}
$$

Certainly this makes sense for mappings in the class $W^{1, n}(X, Y)$.

Now, we have the equipment to do a fair amount of generalizations. If $f$ belongs to the grand Sobolev space $W^{1, n)}(X, Y)$, confront with Definition 2, then

$$
\left.\varphi^{1}=f^{\#} \alpha \in L^{\left.\frac{n}{l}\right)}\left(\wedge^{l} X\right) \cap \operatorname{ker} d \quad \text { and } \quad \varphi^{2}=f^{\#} \beta \in L^{\frac{n}{n-l}}\right)\left(\wedge^{n-l} X\right) \cap \operatorname{ker} d,
$$

as is easy to check. Consequently, the integrals in (28) stay bounded as $\varepsilon$ approaches zero. Note that $W^{1, n)}(X, Y)$ contains the weak- $W^{1, n}(X, Y)$, that is the mappings $f: X \rightarrow Y$ whose generalized differential satisfies the inequality $\operatorname{mes}\{x ;|D f(x)|>t\} \leq C t^{-n}$ for all positive $t$. Unfortunately, we do not know if the limit in (28) actually exists for mappings in the Marcinkiewicz-Sobolev space weak- $W^{1, n}(X, Y)$. In order to get around this delicate question, we denote by $\mathcal{W}^{1, n)}(X, Y)$ the closure of $C^{\infty}(X, Y)$ in the metric of the grand Sobolev space $W^{1, n)}(X, Y)$.

TheOREM 7. Formula (28) is valid for $f \in \mathcal{W}^{1, n)}(X, Y)$. The degree of $f$ is an integer which is invariant under homotopy within the class $\mathcal{W}^{1, n)}(X, Y)$.

As a matter of fact, the degree function $\operatorname{deg}: \mathcal{W}^{1, n)}(X, Y) \rightarrow \mathbb{Z}$ is uniformly continuous. This answers one of the questions in [BN1]. Note too that $\mathcal{W}^{1, n)}(X, Y) \not \subset$ $V M O(X, Y)$. It is not difficult to check that mappings in $\mathcal{W}^{1, n)}(X, Y)$ satisfy

$$
\lim _{\varepsilon \downarrow 0} \varepsilon \int_{X}|D f(x)|^{n-\varepsilon} d x=0,
$$

though we do not know if this is precisely the condition which characterizes the space $\mathcal{W}^{1, n)}(X, Y)$.

One more subclass of $W^{1, n)}(X, Y)$ merits mentioning here, and that is the ZygmundSobolev space of mappings $f: X \rightarrow Y$ such that $\int_{X}|D f|^{n} \log ^{-1}(e+|D f|)<\infty$. We denote it briefly by $\mathcal{Z}(X, Y)$ in remembrance of Antoni Zygmund. That condition (29) holds in this space is undeniable. But the following question remains open.

QUESTION 11. Is the space $C^{\infty}(X, Y)$ dense in $\mathcal{Z}(X, Y)$ ?

Some evidence for it can be found in the recent work of F. Bethuel [Be]. As a rather interesting inference from Theorem 7 , let us assume that $f$ preserves the orientation, that 
is $J(x, f) \geq 0$ a.e. Passing to the limit under the integral sign in (28) is legitimate and yields: $\operatorname{deg}(f ; X, Y)=\int_{X} J(x, f) d x$, though $|D f|^{n}$ may not be integrable. There are also the nagging questions of spheres and manifolds with boundary.

QUeSTION 12. How can we get rid of the unwelcome condition $\mathcal{H}^{l}(Y) \neq 0$ ?

QUESTION 13. How to extend formula (28) to noncompact manifolds?

To this latter question the answer can possibly be reached from [BN2].

Coming to an end, there are a few results and problems along the lines of these lectures which we shall have to postpone. For instance those concerning quasiregular semigroups [IM 4], [T1], [H] or Stoilow's type theorem for mappings with finite distortion [D], [IŠ], [MV], [T2], [HK]. Quasiconformal mappings and the governing PDEs, as evidenced by current literature, attain the status of an important branch of classical analysis. Many fascinating and useful connections with non-linear elasticity, calculus of variations, singular integrals, etc. remain yet to be discovered.

\section{References}

[A] S. S. Antman, Fundamental Mathematical Problems in the Theory of Nonlinear Elasticity, North-Holland, (1976), 33-54.

[AD] J.J. Alibert and B. Dacorogna, An example of a quasiconvex function that is not polyconvex in two dimensions, Arch. Rat. Mech. Anal. 117 (1992), 155-166.

[As] K. Astala, Area distortion of quasiconformal mappings, Acta Math. 173 (1994), $37-60$.

[B] J. M. BALL, Convexity conditions and existence theorems in nonlinear elasticity, Arch. Rat. Mech. Anal. 63 (1977), 337-403.

[Ba] Z. M. BALOGH, Jacobians in Sobolev spaces, preprint.

[Bo] B. Bojarski, Generalized solutions of a system of differential equations of first order and elliptic type with discontinuous coeffieients, Mat. Sbornik 43 (1957), 451-503.

[Be] F. Bethuel, The approximation problem for Sobolev maps between two manifolds, Acta Math. 167 (1991), 153-206.

[BG] L. Boccardo and T. Gallouet, Non linear elliptic and parabolic equations involving measure data, Jour. Func. Anal. 87 (1989), 149-169.

[BBH] F. Bethuel, H. Brezis and F. Helein, Ginzburg-Landau Vortices, Birkhäuser, 19.

[BK] D. Burago and B. Kleiner, Separated nets in Euclidean space and Jacobians of bi-Lipschitz maps, Geom. Funct. Anal. 8 (1998), 273-282.

[BL] R. Bañuelos and A. Lindeman, A martingale study of the Beurling-Ahlfors transform in $R^{n}$, Journal of Funct. Anal. 145 (1997), 224-265.

[BI] B. Bojarski and T. IwANiec, Analytical foundations of the theory of quasiconformal mappings in $R^{n}$, Ann. Acad. Sci. Fenn. Ser. A I Math. 8 (1983), 257-324.

[BM-S] A. Baernstein and S. J. Montgomery-Smith, Some conjectures about integral means of $\partial f$ and $\bar{\partial} f$, preprint.

[BN1] H. Brezis and L. Nirenberg, Degree theory and BMO. Part I: Compact manifolds without boundaries, Selecta Math. 1 (1995), 197-263.

[BN2] H. Brezis and L. Nirenberg, Degree theory and BMO. Part II: Manifolds with boundaries, Selecta Math. 2 (1996), 309-368. 
[BIS] L. Budney, T. Iwaniec and B. Stroffolini, Removability of singularities of Aharmonic functions, Differential and Integral Equations 12 (1999), 261-274.

[Bu] D. Burkholder, Sharp inequalities for martingales and stochastic integrals, Astérisque 157-158, (1988), 75-94.

[BW] R. BAÑUelos and G. WANG, Sharp inequalities for martingales with applications to the Beurling-Ahlfors and Riesz transforms, Duke Math. J. 80 (1995), 575-600.

[CG] R. Coifman and L. Grafakos, Hardy space estimates for multilinear operators I, Revista Matematica Iberoamericana 8 (1992), 45-67.

[CJMR] M. Cwikel, B. Jawerth, M. Milman and R. Rochberg, Differential estimates and commutators in interpolation theory, London Math. Soc. Lecture Notes 138 (1989), $170-220$

[CLMS] R. R. Coifman, P. L. Lions, Y. Meyer and S. Semmes, Compensated compactness and Hardy spaces, J. Math. Pures Appl. (9) 72 (1993), 247-286.

[Da] B. Dacorogna, Direct Methods in the Calculus of Variations, Springer-Verlag, Berlin 1989

[D] G. David, Solutions de l'équation de Beltrami avec $\|\mu\|_{\infty}=1$, Ann. Acad. Sci. Fenn. Ser. A I Math. 13 (1988), 25-70.

[DM] B. DAcorogna and J. Moser, On a partial differential equation involving the Jacobian determinant, Ann. I.H.P. Analyse Non Linéaire 7 (1990), 1-26.

[DHM 1] G. Dolzmann, N. Hungerbühler and S. Müller, Non-linear elliptic systems with measure-valued right hand side, Math. Z. 226 (1997), 545-574.

[DHM 2] G. Dolzmann, N. HungerbüHler and S. Müller, The p-harmonic system with measure-valued right hand side, Ann. Inst. H. Poincaré Anal. Non Linéaire 14 (1997), 353-364.

[DS] S. K. Donaldson and D. B. Sullivan, Quasiconformal 4-manifolds, Acta Math. 163 (1989), 181-252.

[EH] A. Eremenko and D. Hamilton, On the area distortion by quasiconformal mappings, Proc. Amer. Math. Soc. 123 (1995), 2793-2797.

[EM] L. C. EvANS and S. MüLLER, Hardy spaces and the two-dimensional Euler equations with nonegative vorticity, J. Amer. Math. Soc. 7 (1994), 199-219.

[EsM] M. J. Esteban and S. MüLLER, Sobolev maps with integer degree and applications to Skyrme's problem, Proc. Roy. Soc. London 436 A (1992), 197-201.

[G] L. Greco, A remark on the equality det $D f=$ Det $D f$, Diff. Int. Eq. 6 (1993), 1089-1100.

[Ge] F. W. GeHRIng, The $L^{p}$-integrability of the partial derivatives of a quasiconformal mapping, Acta Math. 130 (1973), 265-277.

[GI] F. W. Gehring and T. Iwaniec, The limit of mappings with finite distortion, Ann. Acad. Sci. Fenn. 24 (1999), to appear.

[GIM] L. Greco, T. Iwaniec and G. Moscariello, Limits of the improved integrability of the volume forms, Indiana University Math. J. 44 (1995), 305-339.

[GIS] L. Greco, T. IwANiEC and C. SBordone, Inverting the p-harmonic operator, Manuscripta Mathematica 92 (1997), 249-258.

[GISS] L. Greco, T. Iwaniec, C. Sbordone and B. Stroffolini, Degree formulas for maps with nonintegrable Jacobian, Topological Methods in Nonlinear Analysis 6 (1995), 81-95.

[GMS] J. M. Giaquinta, G. Modica and J. SouČEK, Remarks on the degree theory, J. Funct. Anal. 125 (1994), 172-200. 
[HKM] J. Heinonen, T. Kilpeläinen and O. Martio, Nonlinear Potential Theory of Degenerate Elliptic Equations, Oxford University Press, Oxford 1993.

[HK] J. Heinonen and P. Koskela, Sobolev mappings with integrable dilatation, Arch. Rat. Mech. Anal. 125 (1993), 81-97.

[H] A. HinkKanen, Uniformly quasiregular semigroups in two dimensions, Ann. Acad. Sci. Fenn. Math. 21 (1996), 205-222.

[I 1] T. IwANIEC, p-harmonic tensors and quasiregular mappings, Ann. of Math. 136, (1992) 589-624.

[I 2] T. IwANIEC, Integrability theory of the Jacobians, Lipschitz Lectures, preprint No. 36, Sonderforschungsbereich 256, Bonn 1995, pp. 1-68.

[I 3] T. IWANIEC, Current advances in quasiconformal geometry and nonlinear analysis, Proceedings of the XVIth Rolf Nevanlinna Colloquium, Eds.: Laine/Martio, Walter de Gruyter \& Co. (1996), pp. 59-80.

[I 4] T. IwANIEC, The failure of lower semicontinuity for the linear dilatation, Bull. London Mathematical Society 30 (1998), 55-61.

[I 5] T. IwANIEC, Nonlinear commutators and Jacobians, Lectures in El Escorial (Spain, 1996), special issue of the Journal of Fourier Analysis and Applications dedicated to Miguel de Guzman, Vol. 3 (1997), 775-796.

[I 6] T. IwANIEC, Nonlinear Cauchy-Riemann operators in $\mathbb{R}^{n}$, Proc. AMS, to appear.

[IL 1] T. Iwaniec and A. Lutoborski, Integral estimates for null Lagrangians, Arch. Rat. Mech. Anal. 125 (1993), 25-79.

[IL 2] T. IwANiEC and A. Lutoborski, Polyconvex functionals for nearly conformal deformations, SIAM J. Math. Anal., Vol. 27, No. 3 (1996), pp. 609-619.

[IM 1] T. IWANIEC and G. MARTIN, Quasiconformal mappings in even dimensions, Acta Math. 170 (1993), 29-81.

[IM 2] T. IWANIEC and G. MARTIN, Riesz transforms and related singular integrals, J. reine angew. Math. 473 (1996), 25-57.

[IMNS] T. Iwaniec, L. Migliaccio, L. NAnia and C. Sbordone, Integrability and removability results for quasiregular mappings in high dimensions, Math. Scand. 75 (1994), 263-279.

[IMS] T. Iwaniec, M. Mitrea and C. Scott, Boundary value estimates for harmonic forms, Proc. Amer. Math. Soc. 124 (1996), pp. 1467-1471.

[ISS] T. Iwaniec, C. Scott and B. Stroffolini, Nonlinear Hodge theory on manifolds with boundary, Annali di Matematica pura ed Applicata, to appear.

[IŠ] T. IwanIEC and V. Šverak, On mappings with integrable dilatation, Proc. Amer. Math. Soc. 118 (1993), 181-188.

[IS 1] T. IWANIEC and C. SBORDONE, On the integrability of the Jacobian under minimal hypotheses, Arch. Rat. Mech. Anal. 119 (1992), 129-143.

[IS 2] T. Iwaniec and C. Sbordone, Weak minima of variational integrals, J. Reine Angew. Math. 454 (1994), 143-161.

[IS 3] T. Iwaniec and C. Sbordone, Riesz transforms and elliptic PDEs with VMOcoefficients, Journal d'Analyse Mathématique 74 (1998), 183-212.

[IV 1] T. Iwaniec and A. Verde, A study of Jacobians in Hardy-Orlicz spaces, Proc. Royal Soc. Edinburgh (1999).

[IV 2] T. IwANIEC and A. Verde, Note on the operator $\mathcal{L}(f)=f \log |f|$, submitted to the Journal of Functional Analysis. 
[Ka1] N. J. KALton, Differential methods in interpolation theory, notes of the lectures at the University of Arkansas, April 10-13, 1996.

[Ka2] N. J. KAlton, Nonlinear commutators in interpolation theory, Memoirs AMS 385, vol. 73, (1988), 1-85.

[K] T. KILPELÄINen and J. MALY, Degenerate elliptic equations with measure data and nonlinear potentials, Ann. Scuola Norm. Sup. Pisa 19 (1992), 591-613.

[L] J. LewIs, On very weak solutions of certain elliptic systems, Comm. Partial Differential Equations 18 (1993), 1515-1537.

[Li] A. Lindeman, Martingales and the n-dimensional Beurling-Ahlfors transform, preprint (1996).

[LM] H. B. Lawson and M. L. Michelson, Spin Geometry, Princeton Univ. Press, Princeton, 1989.

[McM] C. T. McMullen, Lipschitz maps and nets in Euclidean space, Geom. Funct. Anal. 8 (1998), 304-314.

[Mi] M. Milman, Higher order commutator in the real method of interpolation, Journal D'Analyse Math. 66 (1995), 37-56.

[M] J. Moser, On the volume elements on a manifold, Trans. Amer. Math. Soc. 120 (1965), 286-294.

[MR] M. Milman and R. Rochberg, The role of cancellation in interpolation theory, Contemporary Math. 189 (1995), 403-410.

[MS] M. Milman and T. Schonbek, A note on second order estimates in interpolation theory and applications, Proc. AMS 110 (1990), 961-969.

[Mu1] F. Murat, Compacité par compensations, Ann. Sc. Norm. Sup. Pisa 5 (1978), 489-507.

[Mu2] F. Murat, Soluciones renormalizades de EDP elipticas no linear, Publications du Laboratoire d'Analyse Numerique, Paris (1993).

[Ma] J. MANFREDI, Quasiregular mappings from the multilinear point of view, Ber. University, Jyväskylä Math. Inst. 68 (1995), 55-94.

[MV] J. MANFredi and E. Villamor, Mappings with integrable dilatation in higher dimensions, Bull. Amer. Math. Soc. 32 (1995), 235-240.

[Mü] S. MÜLLER, A surprising higher integrability property of mappings with positive determinant, Bull. Amer. Math. Soc. 21 (1989), 245-248.

[R] H. M. Reimann, Harmonische Funktionen und Jacobi-Determinanten von Diffeomorphismen, Comment. Math. Helv. 47 (1972), 397-408.

[RRT] J. W. Robbin, R. C. Roger and B. Temple, On weak continuity and the Hodge decomposition, Trans. AMS 303 (1987), 609-618.

[RY] T. RIVERE and D. YE, Resolutions of the prescribed volume form equation, Nonlinear Differential Equations Appl. 3 (1996), 323-369..

[Ri] S. Rickman, Quasiregular Mappings, Springer-Verlag, Berlin 1993.

[Re 1] Y. G. RESHETNYAK, On extremal properties of mappings with bounded distortion, Sibirsk. Mat. Z. 10 (1969), 1300-1310 (Russian).

[Re 2] Y.G. Reshetnyak, Space Mappings with Bounded Distortion, Trans. Math. Monographs 73, Amer. Math. Soc., 1989.

[Ro] R. RochBerG, Higher order estimates in complex interpolation theory, Pacific J. Math. 174 (1996), 247-267.

[RW] R. Rochberg and G. WeIss, Derivatives of analytic families of Banach spaces, Ann. of Math. 118 (1983), 315-347. 
[S] E. M. Stein, Note on the class LlogL, Studia Math. 32 (1969), 305-310.

[Sc] C. Scott, $L^{p}$-theory of differential forms on manifolds, Trans. Amer. Math. Soc. 347 (1995), 2075-2096.

[Se] S. Semmes, A primer on Hardy spaces, and some remarks on a theorem of Evans and Müller, Commun. in PDEs 19 (1994), 277-319.

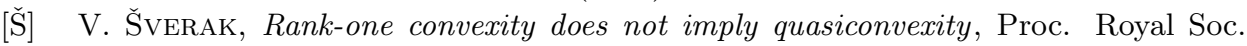
Edinburgh 120A (1992), 185-189.

[Ta] L. TARTAR, Compensated compactness and applications to partial differential equations in Nonlinear Analysis and Mechanics, Heriotwatt Symposium IV, Research Notes in Mathematics, R. J. Knops (ed.), vol. 39, Pitman, London, (1979), 136-212.

[T 1] P. TUkIA, On quasiconformal groups, J. Analyse Math. 46 (1986), 318-346.

[T 2] P. TukIA, Compactness properties of $\mu$-homeomorphisms, Ann. Acad. Sci. Fenn. Ser. A I Math. 16 (1991) 47-69.

[V 1] J. VÄISÄLÄ, Lectures on n-Dimensional Quasiconformal Mappings, Lecture Notes in Mathematics, 229, Springer-Verlag, 1971.

[V 2] J. VÄıs̈̈LÄ, Questions on quasiconformal maps in space, in: Quasiconformal Mappings and Analysis, Springer, New York, 1998, 369-374.

[VG] S. K. Vodop'Yanov and V. M. Goldstein, Quasiconformal mappings and spaces of functions with generalized first derivatives, Sibirsk. Mat. Z. 17 (1976), 515-531 (Russian).

[Vu] M. VuORInen (ed.), Quasiconformal Space Mappings, A collection of Surveys 19601990 (Lecture Notes in Math. 1508), Springer-Verlag, Berlin 1992.

[W] W. Wente, An existence theorem for surfaces of constant mean curvature, J. Math. Anal. Appl. 26 (1969), 318-344.

[Ye] D. Ye, Prescribing the Jacobian determinant in Sobolev spaces, Ann. Inst. H. Poincaré Anal. Non Linéaire 11 (1994), 275-296. 